\title{
CURRENT-CONTROLLED CURRENT DIFFERENCING BUFFERED AMPLIFIER: IMPLEMENTATION AND APPLICATIONS
}

\author{
SUDHANSHU MAHESHWARI* and IQBAL A. KHAN \\ Department of Electronics Engineering, Zakir Hussain College of Engineering and \\ Technology, Aligarh Muslim University, Aligarh 202 002, India
}

(Received 29 October 2003; In final form 17 November 2003)

\begin{abstract}
A new four terminal current-controlled active element is introduced, where parasitic resistances at two current input ports are controlled leading to the definition of current-controlled current differencing buffered amplifier. Bipolar implementation and as application current-mode band-pass filter circuits are proposed. Simulation results using real device parameters are included, which show device bandwidth of $35 \mathrm{MHz}$, low total harmonic distortions, and tuning over a wide current range.
\end{abstract}

Keywords: Current mode circuits; Band-pass filters

\section{INTRODUCTION}

Recently current-mode circuits have gained popularity because of their potential advantages such as wider bandwidths, higher slew rates, greater linearity, wider dynamic range, and simple circuitry [1-4]. Several current-mode applications have thus been reported using a number of active elements [5-9]. Tunable electronic functions have also been realized using current-controlled conveyors (CCCII) with external current control $[6,10,11]$. Current differencing buffered amplifier (CDBA), a relatively new active element, offers the advantage of high impedance current output as well as a buffered voltage output [7]. The finite parasitic resistance at the two input ports of the CDBA (P and N) can be used as an advantage, if made controllable. In this article, these resistances are current-controlled using mixed translinear input loops and current mirrors so as to lead to the definition of current-controlled current differencing buffered amplifier (CCCDBA). As an application, a current-mode band-pass filter circuit is given and the circuit is tuned using the parasitic port resistances through external current control. SPICE simulation results using real circuit parameters of transistors NR100N and PR100N [12] are also given.

\footnotetext{
* Corresponding author. E-mail: sudhanshu_maheshwari@rediffmail.com
} 


\section{DEVICE PROPOSED}

The already reported CDBA is characterized by the following port relationship [7].

$$
V_{p}=V_{n}=0, \quad I_{z}=I_{p}-I_{n}, \quad V_{w}=V_{z} .
$$

Here the two input terminals ( $\mathrm{P}$ and $\mathrm{N}$ ) offer a finite parasitic resistance [6]. These parasitic resistances can be current-controlled in a CCCDBA whose symbol and bipolar implementation are given in Figure 1. The circuit implementation of Figure 1(b) consists of mixed translinear loops [13] (transistors 1-6) realizing two input terminals with a ground potential. The mixed loops are dc biased by $I_{\mathrm{o}}$ using mirrors (transistors 7-13). The output Z-terminal that generates the current difference of P- and N-terminals is realized using transistors (14-21). The buffered output $(W)$ is realized using transistors (22-25). The P- and N-terminal resistance can be obtained by the following relationship [6].

$$
R_{p, n}=\frac{V_{\mathrm{T}}}{2 I_{\mathrm{o}}}
$$
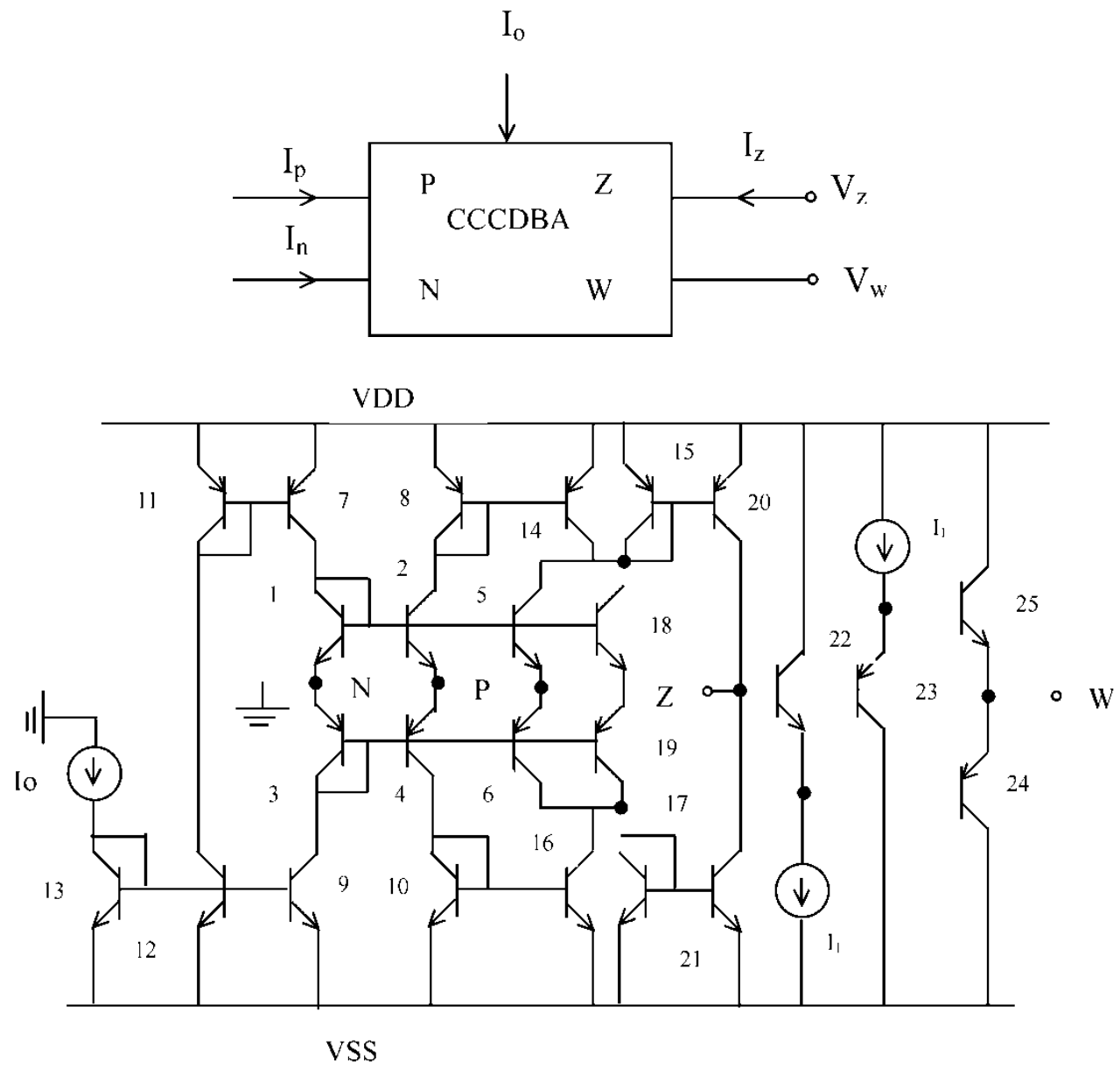

FIGURE 1 (a) CCCDBA symbol and (b) implementation of CCCDBA. 
From Eq. (2), it is clear that the P- and N-terminal resistances can be varied by external bias current $\left(I_{\mathrm{o}}\right)$ of the CCCDBAs. It is to be noted that the proposed implementation of CCCDBA is suited for IC implementation by replacing the current sources used in the buffer stage by transistorized circuitry. A simple arrangement for the purpose is to use the basic two transistor current source and a steering transistor to obtain two equal currents.

\section{APPLICATION EXAMPLES}

Using the introduced active element, a current-mode band-pass filter (BPF1) is realized and shown in Figure 2(a). It is to be noted that the circuit of Figure 2(a) is based on the $R-L-C$ parallel resonator, where the inductor is realized using the CCCDBAs and capacitor $C_{1}$ $\left(L=R_{n 1} R_{p 2} C_{1}\right)$. One such inductance simulator employing CDBAs and controllable metal oxide semiconductor (MOS) resistors is available in literature [14]. The circuit used here for the inductance simulator enjoys electronic tuning as a result of new controlled active element (CCCDBA). The resulting inductance simulator is controllable over a wide range by varying the bias current of the CCCDBA. Routine analysis of the circuit of Figure 2(a) yields the following current transfer function.

$$
\frac{I_{\mathrm{out}}}{I_{\mathrm{in}}}=\frac{s / R C_{2}}{s^{2}+s / R C_{2}+1 / R_{p 2} R_{n 1} C_{1} C_{2}} .
$$
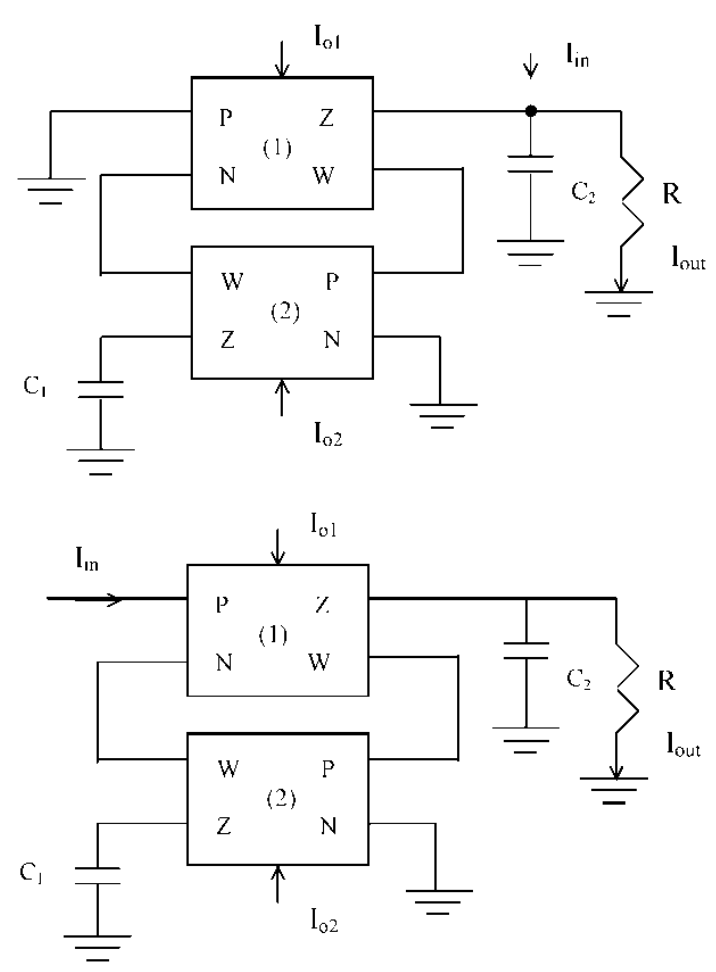

FIGURE 2 (a) Current-mode BPF1 using CCCDBAs and (b) current-mode BPF2 using CCCDBAs. 
It is to be noted that the circuit simultaneously provides two additional current transfer functions given as:

$$
\begin{aligned}
\frac{I_{C 1}}{I_{\text {in }}} & =\frac{\left(R / R_{p 2}\right) s / R C_{2}}{s^{2}+s / R C_{2}+1 / R_{p 2} R_{n 1} C_{1} C_{2}} \\
\frac{I_{C 2}}{I_{\text {in }}} & =\frac{s^{2}}{s^{2}+s / R C_{2}+1 / R_{p 2} R_{n 1} C_{1} C_{2}} .
\end{aligned}
$$

Here, $R_{p 2}$ is the P-terminal resistance of CCCDBA(2) and $R_{n 1}$ is the N-terminal resistance of CCCDBA(1). It is clear that the pole- $\mathrm{w}_{\mathrm{o}}$ of the band-pass filter can be adjusted by varying the parasitic resistance with external bias currents of the CCCDBAs. As compared to the bandpass filter based on CCCIIs of Ref. [6], the new active element based circuit offers independent tuning of filter pole- $\mathrm{w}_{\mathrm{o}}$ and pole-Q. Similarly, when compared to another recently reported circuit using CCCIIs, the new band-pass filter enjoys the orthogonal tuning of pole- $\mathrm{w}_{\mathrm{o}}$ and pole-Q and unity pass band gain. However, the reported work has the advantage of high output impedance [15]. It is also to be noted that a high-pass filter is simultaneously realized across $C_{2}$ and an additional band-pass filter is obtained across $C_{1}$, a pass-band gain $R / R_{p 2}$. These features are additional to the above-mentioned advantage of the circuit though the circuit complexity of CCCDBA is more as compared to the CCCIIs used in other works $[6,15]$. The circuit complexity, and hence the silicon area required for IC realizations of the proposed band-pass filter, is better compared to one of the excellent reported band-pass filters with orthogonal control of filter parameters employing four CCCIIs and three passive components [16]. The proposed circuit is found to be as compact as the reported application work.

Next, some band-pass filter circuits are introduced that better utilize the current differencing property of the new active element CCCDBA. One such band-pass filter circuit (BPF2) is shown in Figure 2(b) and has the current transfer function the same as Eq. (3). The other two current transfer functions are also found the same as given by Eqs. (4) and (5). It is further to be noted that another band-pass filter circuit (BPF3) can be obtained if $R$ is connected to the Z-terminal of CCCDBA(2) instead of CCCDBA(1) and current inputted at the $\mathrm{N}$-terminal of CCCDBA(2), P of CCCDBA(1) being grounded. The output across $R$ and $C_{2}$ then gives band-pass responses with unity gain and $R / R_{n 1}$ gain, respectively. Another response, namely high pass, is obtained if current through $C_{1}$ is sensed. Now in this configuration if current is inputted at $\mathrm{P}$ of $\operatorname{CCCDBA}(1), \mathrm{N}$ of $\operatorname{CCCDBA}(2)$ grounded, then the resulting circuit (BPF4) realizes the band-pass and low-pass functions through $C_{1}$ and $R$, respectively. These applications further confirm the utility of the CCCDBAs.

\section{NON-IDEAL EFFECTS}

The non-ideal CCCDBA is characterized by $I_{z}=\left(\alpha_{p} I_{p}-\alpha_{n} I_{n}\right)$, where $\alpha_{p}$ and $\alpha_{n}$ deviate from unity by the transfer errors and $V_{w}=\beta V_{z}$, where $\beta$ deviates from unity by the voltage transfer error. The BPF1 of Figure 2(a) is analyzed for these non-idealities of the CCCDBA and the transfer functions given as:

$$
\frac{I_{\text {out }}}{I_{\text {in }}}=\frac{s / R C_{2}}{s^{2}+s / R C_{2}+\alpha_{n 1} \alpha_{p 2} \beta_{1} \beta_{2} / R_{p 2} R_{n 1} C_{1} C_{2}} .
$$


Similarly Eqs. (4) and (5) are modified as:

$$
\begin{aligned}
& \frac{I_{C 1}}{I_{\text {in }}}=\frac{\alpha_{2} \beta_{1}\left(R / R_{p 2}\right) s / R C_{2}}{s^{2}+s / R C_{2}+\alpha_{n 1} \alpha_{p 2} \beta_{1} \beta_{2} / R_{p 2} R_{n 1} C_{1} C_{2}} \\
& \frac{I_{C 2}}{I_{\text {in }}}=\frac{s^{2}}{s^{2}+s / R C_{2}+\alpha_{n 1} \alpha_{p 2} \beta_{1} \beta_{2} / R_{p 2} R_{n 1} C_{1} C_{2}} .
\end{aligned}
$$

Here, indexes 1 and 2 stand for CCCDBA1 and CCCDBA2, respectively. It is seen from Eqs. (6)-(8) that the pole- $\mathrm{w}_{\mathrm{o}}$ is slightly affected as a result of non-unity current and voltage transfer gains. For $I_{C 1}$ the pass-band gain is also affected. However, the sensitivities of pole- $\mathrm{w}_{\mathrm{o}}$ and pole-Q to transfer gain are found to be 0.5 in magnitude and the sensitivity of filter gain for $I_{C 1}$ to these transfer gains is within unity in magnitude that represents a low value. Thus, the circuit enjoys good sensitivity performance. Similarly the other BPFs of Figures 2(b) and (3) are also found to exhibit good performance after accounting for the non-idealities. It is to be noted that the transfer gains are frequency-dependent and shall also become apparent from their frequency responses to be given later.

Another source of non-ideality are the parasitic impedances at various ports. The finite parasitic resistances at $\mathrm{P}$ - and $\mathrm{N}$-terminals have already been used to advantage and are
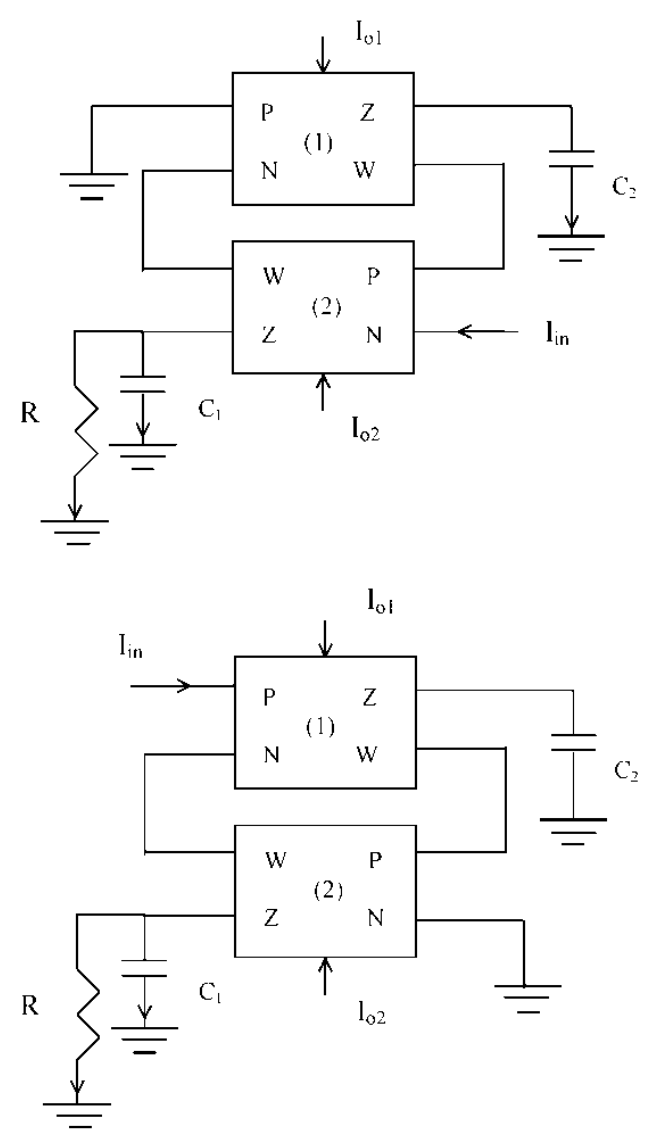

FIGURE 3 (a) Current-mode BPF3 using CCCDBAs and (b) current-mode BPF4 using CCCDBAs. 
key to the new controlled active element. The effect of parasitic capacitance at Z-terminals $\left(C_{z}\right)$ is minimized since capacitors are employed in the proposed circuit configuration at those terminals of both the CCCDBAs.

\section{SIMULATION RESULTS}

The proposed CCCDBA is simulated using transistor NR100N and PR100N parameters [12] with supply voltage $\pm 2.5 \mathrm{~V}$. The short circuit current gain of the CCCDBA is shown in Figure 4(a) that shows a bandwidth of $35 \mathrm{MHz}$. Similarly, the output voltage $\left(V_{w}\right)$ is measured with a load of $1 \mathrm{kohm}$ at the Z-terminal [Fig. 4(b)] and an equal bandwidth obtained for a bias current $I_{\mathrm{o}}=100 \mu \mathrm{A}$ in both cases. The variation of port resistances $\left(R_{p, n}\right)$ with the bias current of CCCDBA is shown in Figure 5, which shows a wide variation and good matching between theoretical ' $R_{p, n \text { (th) }}$ ' and measured values ' $R_{p, n \text { (pr) }}$ '. Thus, the realizations based on the new CCCDBA would enjoy a wide range of electronic tuning by varying the bias current. The CCCDBA is also studied for harmonic distortions. The total harmonic distortions (THD) for the proposed active element are measured at the output terminal by inserting sinusoidal current inputs at the P- and N-terminals. The THD results obtained for $R_{p, n}$ are tabulated in Table I and show low THD for two different frequencies $(100 \mathrm{kHz}$ and $1 \mathrm{MHz})$ for varying values of the bias currents. Next the CCCDBA is biased at $I_{\mathrm{o}}=100 \mu \mathrm{A}$ and $100 \mathrm{kHz}$ sinusoidal inputs are applied at the input terminals such that $I_{p}=0.1 \mathrm{~mA}$ constant

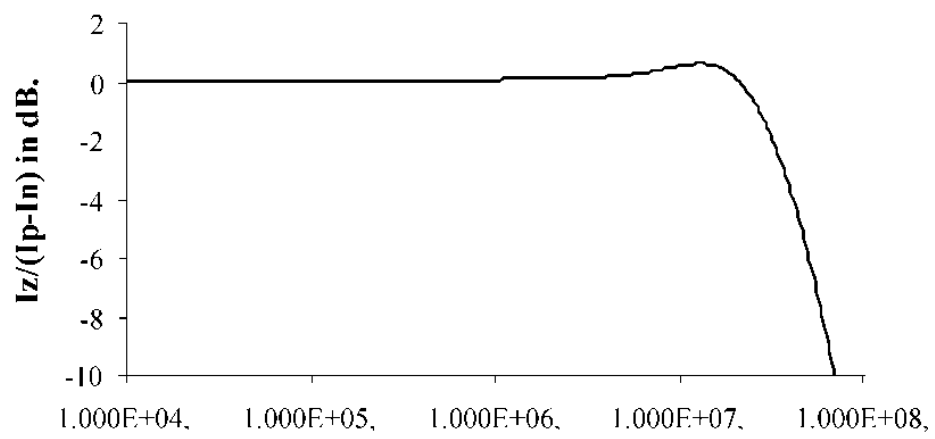

Frequency (Hz.)

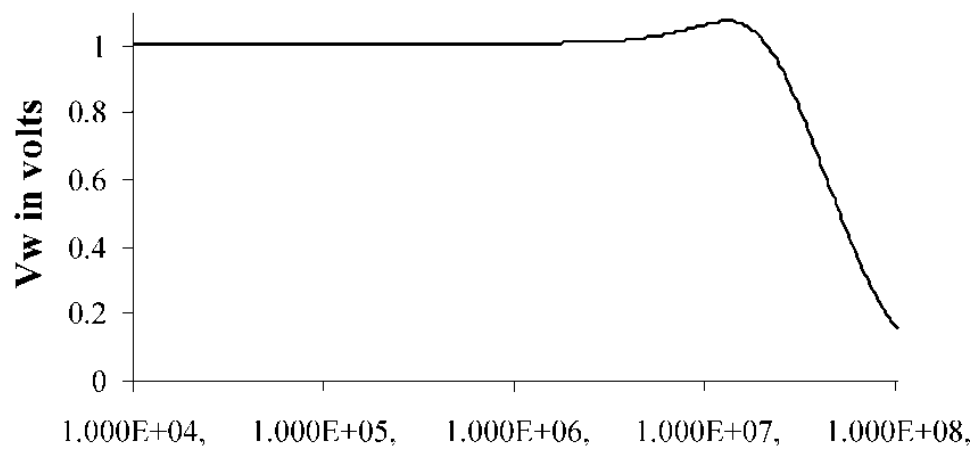

Frequency (Hz.)

FIGURE 4 (a) Short circuit current gain of CCCDBA and (b) frequency characteristics of buffered output. 


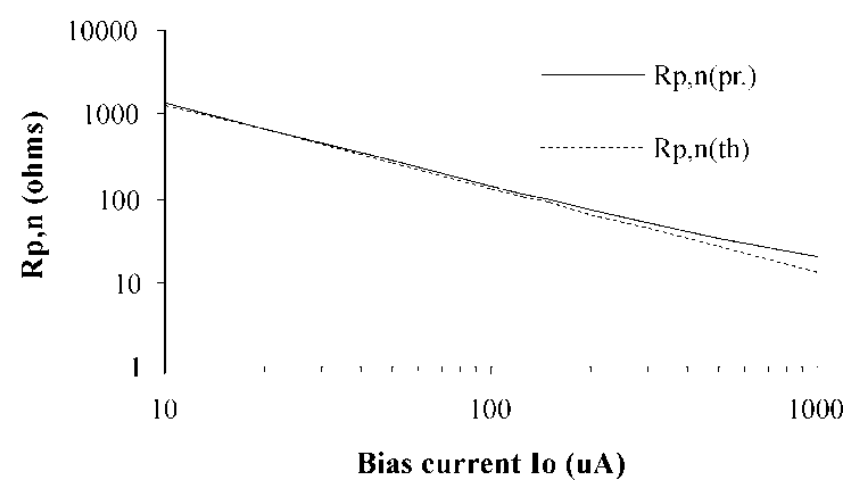

FIGURE 5 Variation of parasitic resistance $\left(R_{p, n}\right)$ with bias current $\left(I_{\mathrm{o}}\right)$.

TABLE I The THD for Varying Bias Currents of $R_{p, n}$.

\begin{tabular}{lccccr}
\hline$I_{\mathrm{o}}(\mu \mathrm{A})$ & 10 & 100 & 200 & 500 & 1000 \\
THD $(\%)$ at $100 \mathrm{kHz}$ & 0.96 & 0.76 & 0.46 & 0.26 & 0.13 \\
THD $(\%)$ at $1 \mathrm{MHz}$ & 1.9 & 0.73 & 0.60 & 0.40 & 0.27 \\
\hline
\end{tabular}

TABLE II The THD at $I_{\mathrm{o}}=100 \mu \mathrm{A}, 100 \mathrm{kHz}$ Sinusoidal Inputs to CCCDBA for Varying Magnitude of $I_{n}$ and $I_{p}=0.1 \mathrm{~mA}$ constant.

\begin{tabular}{lllllll}
\hline$I_{p}(\mathrm{~mA})$ & 0.2 & 0.5 & 0.8 & 1.0 & 2.0 & 5.0 \\
THD $(\%)$ & 0.33 & 0.47 & 0.61 & 0.69 & 0.70 & 1.08 \\
\hline
\end{tabular}

and for varying magnitudes of $I_{n}$. The THD obtained are tabulated in Table II and show low values. It is thus concluded that the CCCDBA also enjoys good THD performance.

The current-mode band-pass filter of Figure 2 (a) is next designed for varying pole- $\mathrm{w}_{\mathrm{o}}$. The designed values used are $C_{1}=C_{2}=10 \mathrm{nF}, R=1 \mathrm{kohm} . I_{\mathrm{o} 1}$ and $I_{\mathrm{o} 2}$ are varied from 100 to $150 \mu \mathrm{A}$ in steps of $25 \mu \mathrm{A}$ to obtain frequency tuning in the range from 100 to $150 \mathrm{kHz}$. The center frequency tuning of the current mode band-pass filter circuit by bias current variation of CCCDBAs is shown in Figure 6 and verifies the BPF1. Next, the circuit of Figure 3(a) (BPF3) is also designed for center frequency $100 \mathrm{kHz}$. The designed values

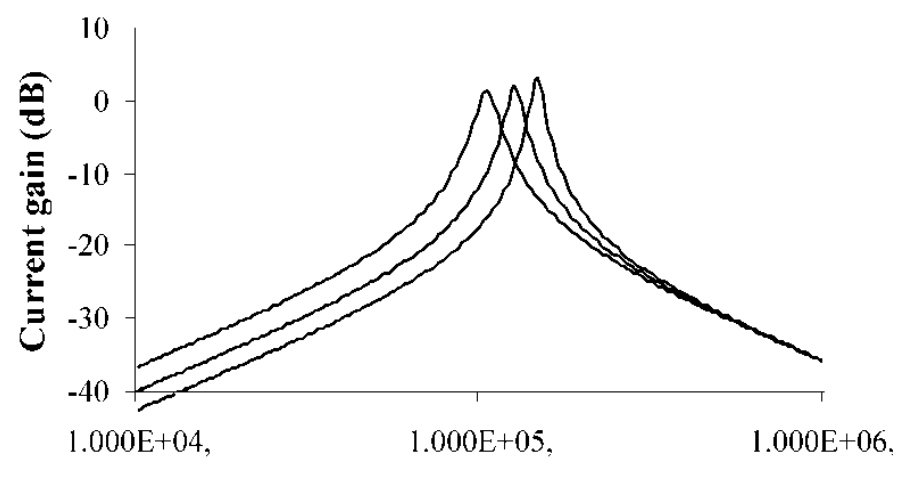

Frequency $(\mathrm{Hz})$

FIGURE 6 Frequency response of BPF1 showing pole- $w_{\mathrm{o}}$ tuning. 


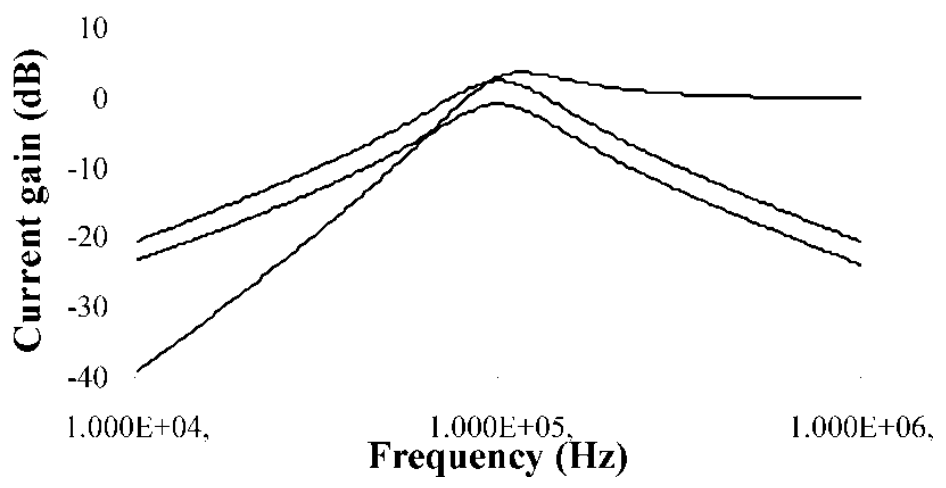

FIGURE 7 Frequency response of BPF3 showing two band-pass and a high-pass response.

are $C_{1}=C_{2}=10 \mathrm{nF}, I_{\mathrm{o} 1}=I_{\mathrm{o} 2}=100 \mu \mathrm{A}$ and $R=0.25 \mathrm{kohm}$. The pole-Q with the designed values is approximately 1.7 . The frequency response of the circuit as shown in Figure 7 depicts the two band-pass outputs and the high-pass output, thus verifying the BPF3 circuit.

\section{CONCLUSION}

New CCCDBA is introduced along with its bipolar implementation. As application, currentmode band-pass filter circuits are shown realized with the new controlled element. The CCCDBA enjoys large bandwidth, low THDs, electronic tuning over a wide range with external current and shows promising results using real device parameters. The new controlled active element is suited for IC implementation and active network synthesis applications.

\section{References}

[1] Wilson, B. (1990). Recent developments in current conveyors and current-mode circuits. IEE Proceedings G., 137, 61-77.

[2] Toumazou, C., Lidgey, F. J. and Waigh, D. S. (1990). Analogue IC Design: The Current Mode Approach, IEE Press.

[3] Sedra, A. S. and Smith, K. C. (1970). A second generation current conveyor and its applications. IEEE Transactions Circuit Theory, 132-134.

[4] Sedra, A. S., Roberts, G. W. and Gohh, F. (1990). The current conveyor: History, progress and new results. IEE Proceedings $G, \mathbf{1 3 7}, 78-87$.

[5] Higashimura, M. and Fukui, Y. (1990). Realisation of current-mode all-pass networks using a current conveyor. IEEE Transactions on Circuits Systems, 660-661.

[6] Fabre, A., Saaid, O. and Boucheron, C. (1995). Current controlled bandpass filter based on translinear conveyors. Electronics Letters, 31, 1727-1728.

[7] Ozoguz, S., Toker, A. and Acar, C. (1999). Current mode continuous time fully integrated universal filter using CDBAs. Electronics Letters, 35, 97-98.

[8] Soliman, A. M. (1999). Theorems relating to port interchange in current mode CCII circuits. International Journal of Electronics, 82, 585-604.

[9] Maheshwari, S. and Khan, I. A. (2001). Novel first order all-pass sections using a single CCIII. International Journal of Electronics, 88, 773-778.

[10] Maheshwari, S. (2002). High CMRR wide bandwidth instrumentation amplifier using current controlled conveyors. International Journal of Electronics, 89, 889-896.

[11] Abuelma'atti, M. T. and Alqahtani, M. A. (1998). A new current controlled multiphase sinusoidal oscillator using translinear conveyors. IEEE Transactions on circuits and systems-II, 45, 881-885.

[12] Frey, D. R. (1993). Log domain filtering: an approach to current mode filtering. IEE Proceeding G, Circuits devices and systems, 140, 406-416.

[13] Fabre, A. (1983). Dual translinear voltage/current converter. Electronics Letters, 19, 1030-1031. 
[14] Toker, A., Ozoguz, S. and Acar, C. (2000). CDBA based fully integrated gyrator circuit suitable for electronically tunable inductance simulation. Int. Journal of Electronics and Communication (AEU)' 54, 293-296.

[15] Minaei, S., Cicekoglo, O., Kuntman, H. and Turkoz, S. (2001). High output impedance current-mode low-pass, band-pass and high-pass filters using current controlled conveyors. International Journal of Electronics, 88, 915-922.

[16] Fabre, A., Saaid, O. and Boucheron, C. (1997). Low power current-mode second order band-pass IF filter. IEEE Transactions on Circuits and Systems-II: Analog and Digital Signal Processing, 44, 436-446. 

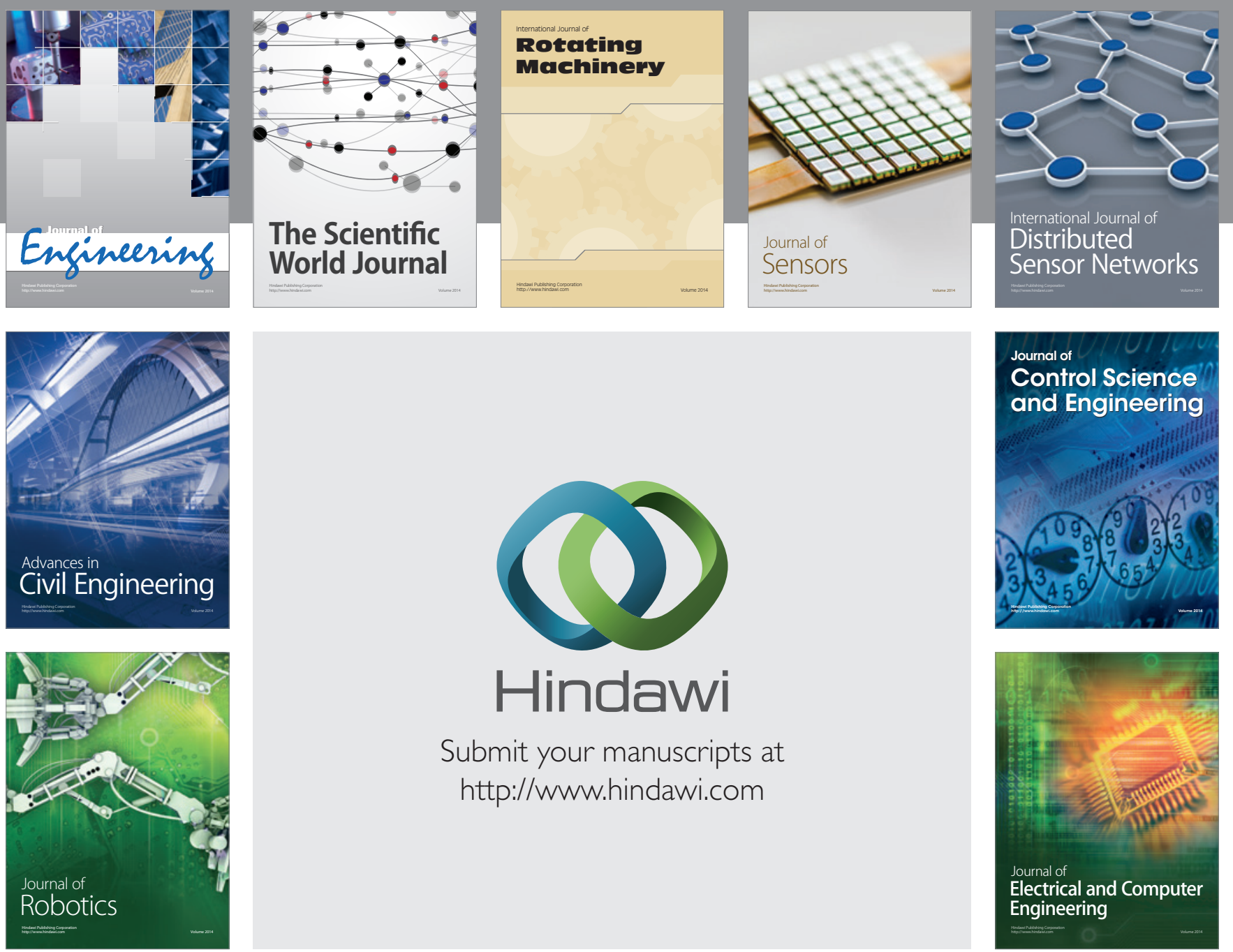

Submit your manuscripts at

http://www.hindawi.com
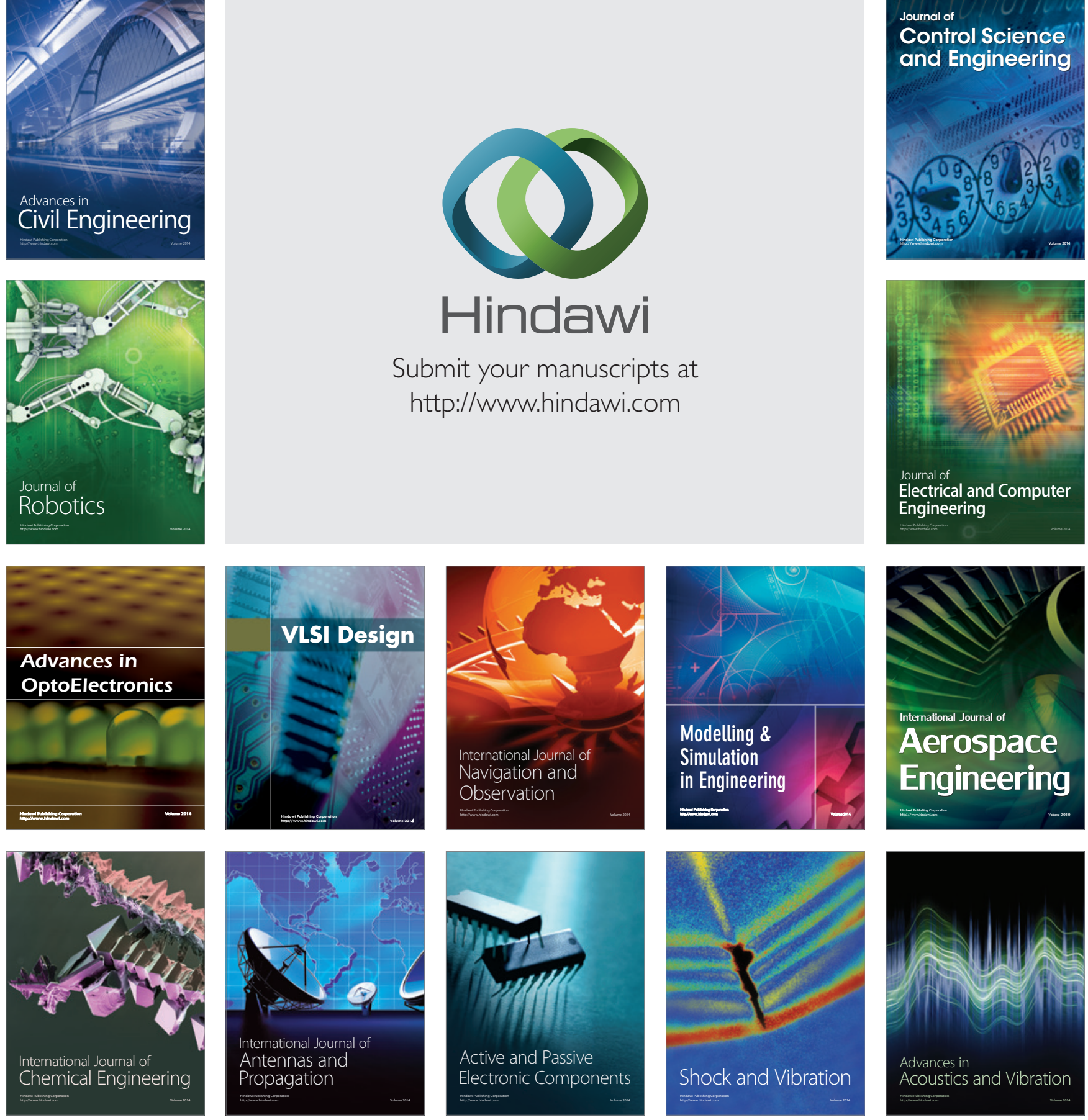